MANLIO SODI

Università Lateranense, Roma

Saeculum Christianum

GIACOMO BAROFFIO

t. XXII (2015), s. 261-279

Università di Pavia, Pavia

ALESSANDRO TONIOLO

\title{
LA CONCORDANTIA DEI TRE GRANDI SACRAMENTARI: GREGORIANO, VERONESE E GELASIANO
}

Tra la fine del II e l'inizio del III millennio la storia del Missale Romanum è stata caratterizzata, oltre che da numerosi studi, anche dall'elaborazione di uno strumento indispensabile per una sua conoscenza più approfondita: la Concordantia.

Nel 1962 è stata pubblicata l'ultima editio typica del Missale Romanum scaturito dalla riforma liturgica voluta dal Concilio Ecumenico Tridentino'; l'anno successivo è apparsa la Concordantia curata da A. Pflieger ${ }^{2}$. Nel 1975 è stata pubblicata l'editio typica altera (secunda) del Missale Romanum - apparso come editio typica nel 1970 - scaturito dalla riforma liturgica voluta dal Concilio Ecumenico Vaticano $\mathrm{II}^{3}$. A partire da questo testo è stata elaborata una Concordantia a cura di T.A. Schnitker e W.A. Slaby ${ }^{4}$.

Questi due "strumenti di lavoro" sono risultati quanto mai preziosi per facilitare la conoscenza dei valori racchiusi nel Missale; e rimangono ancora come punti imprescindibili per chiunque voglia accostarsi a quelle edizioni. D'altra parte, permettono pure di verificare il percorso che caratterizza la storia, l'evoluzione e la trasmissione delle formule del liber princeps della liturgia di rito romano.

\footnotetext{
1 Missale Romanum ex decreto Ss. Concilii Tridentini restitutum, Summorum Pontificum cura recognitum, Editio typica, Typis Polyglottis Vaticanis [s.d. - 1962], pp. LXXXIII + $727+$ [282]. Una ristampa anastatica è apparsa senza data - nel 2011, con la sostituzione dell'orazione contestata della preghiera per gli Ebrei, ma confermando il titolo originario: n. 8. Pro conversione Iudceorum (pag. 173). - Per la storia e l'evoluzione di questo Missale cf M. Sodi - A.M. Triacca (edd.), Missale Romanum. Editio princeps (1570). Edizione anastatica, Introduzione e Appendice $=$ Monumenta Liturgica Concilii Tridentini 2, Lev, Città del Vaticano 1998, ${ }^{2} 2012$, pp. XLVI + 720; M. Sodi - A. Toniolo, Missale Romanum. Editio typica 1962. Edizione anastatica e Introduzione = Monumenta Liturgica Piana 1, Lev, Città del Vaticano 2007, pp. XVIII + 1096. Nel V volume della medesima collana si trova, tra l'altro, la Concordantia elaborata con gli stessi criteri dell'attuale: cf M. Sodi - A. Toniolo - P. Bruylants (edd.), Liturgia tridentina. Fontes - Indices - Concordantia 1568-1962, Lev, Città del Vaticano 2010, pp. XIX + 1254. - Infine va tenuto presente il fascicolo monografico di Rivista Liturgica 95/1 (2008) dal titolo: Celebrare con il Messale di san Pio $V$.

2 Cf A. Pflieger, Liturgica orationis concordantia verbalia. Prima Pars: Missale Romanum, Herder, Romae MCMLIV, pp. XI + 740 .

3 Cf rispettivamente Missale Romanum ex decreto Sacrosancti Ecumenici Concilii Vaticani II instauratum, auctoritate Pauli PP. VI promulgatum, Editio typica, Typis Polyglottis Vaticanis MCMLXX, pp. 966; Editio typica altera MCMLXXV, pp. 999.

4 Cf T.A. Schnitker - W.A. Slaby (edd.), Concordantia verbalia Missalis Romani. Partes euchologicae, Aschendorff, Münster 1983, pp. XV + 1508 (per un totale di 3048 colonne). Cf l'ampia "nota bibliografica" di M. Sodi, Una concordanza verbale del "Missale Romanum” di Paolo VI, in Rivista Liturgica 71/3 (1984) 424-436.
} 
La pubblicazione dell'editio typica tertia del Missale Romanum ${ }^{5}$ ha comportato l'urgenza di uno strumento che desse la possibilità di conoscerne meglio i contenuti, anche in vista della sua "traduzione" nelle lingue nazionali 6 .

L'attuale Missale rinvia però a varie altre fonti che costituiscono il patrimonio di quella traditio che proprio nel Missale realizzato dopo il Vaticano II ha trovato una progressio. Questo è possibile dimostrare solo attraverso una conoscenza attenta delle fonti, e la Concordantia ne è uno strumento privilegiato. In questa linea, pertanto, presentiamo la Concordantia del sacramentario Gregoriano, Veronese e Gelasiano in quanto sono i testimoni più importanti della stessa traditio, e i loro testi sono ampiamente presenti negli attuali libri liturgici ${ }^{7}$.

\section{Le concordanze dei sacramentari}

Da oltre un millennio il patrimonio eucologico dei libri liturgici alimenta la fede della Chiesa. È il fecondo intreccio di relazioni evidenziate con lapidaria chiarezza da Prospero di Aquitania († dopo il 455), quando pone in stretta connessione la lex orandi con la lex credend $i^{8}$. Le orazioni dei grandi sacramentari ${ }^{9}$, e degli altri libri liturgici, rivelano l'elaborazione orante delle riflessioni teologiche ${ }^{10}$ e sollecitano parimenti ulteriori approfondimenti e chiarificazioni ${ }^{11}$.

\footnotetext{
5 Missale Romanum ex decreto Sacrosancti CEcumenici Concilii Vaticani II instauratum, auctoritate Pauli PP. VI promulgatum, Ioannis Pauli PP. II cura recognitum, Editio typica tertia, Typis Vaticanis MMII, pp. 1318. Il Decretum di approvazione porta la data del 20 aprile 2000! Cf il fascicolo monografico di Rivista Liturgica 90/4 (2003) dal titolo: Missale Romanum. La novitas della terza edizione latina.

6 Sulla problematica relativa alle traduzioni cf i fascicoli monografici di Rivista Liturgica 85/6 (1998) sul tema: Oltre la "traduzione"?; 92/1 (2005) sul tema: Quale traduzione per una «liturgia autentica»?; e 92/2 (2005) sul tema: Tradurre testi liturgici: tra sfide e attese. Un testo simile si può leggere anche in Latinitas NS 2/1 (2014) 105-118 sotto il titolo: Tradurre testi per la liturgia: difficoltà, sorprese, prospettive. - In un volume a sé è racchiusa invece la Concordantia della Institutio Generalis Missalis Romani (Editio typica tertia): si tratta di un'opera che raccoglie Textus et Concordantia (oltre ad altri elementi utili), in modo da avere una panoramica completa dei contenuti racchiusi nella parte introduttiva del Missale: cf M. Sodi - A. Toniolo (edd.), Prcenotanda Missalis Romani (Editio typica tertia). Textus - Concordantia - Appendices = Monumenta Studia Instrumenta Liturgica 24, Lev, Città del Vaticano 2002, pp. XIV + 807.

7 Per una panoramica sugli attuali libri liturgici cf il fascicolo monografico di Rivista Liturgica 95/5 (2008) sotto il titolo: La liturgia di rito romano e $i$ suoi libri. - Le tre concordanze sono apparse nella collana „Veterum et Coaevorum Sapientia“ [ = VCS] edita dalla Editrice LAS: M. Sodi - G. Baroffio - A. Toniolo (edd.), Sacramentarium Gregorianum. Concordantia $=$ VCS 7, Roma 2012; Sacramentarium Veronense. Concordantia $=$ VCS 10, Roma 2013; Sacramentarium Gelasianum. Concordantia = VCS 11, Roma 2014.

8 P. D’Aquitania, Indiculus de gratia Dei (cf Denzinger-Schönmetzer 238-242:... ut legem credendi lex statuat supplicandi). Sulla fede trinitaria e il consensus fidelium $\mathrm{cf}$ B. Studer, Lex orandi - lex credendi: Der Taufglaube im Gottesdienst der Alten Kirche, in E. Campi et Al. (edd.), Oratio. Das Gebet in patristischer und reformatorischer Sicht = Forschungen zur Kirchen- und Dogmengeschichte 76, Vandenhoeck et Ruprecht, Göttingen 1999, pp. 139-149.

9 Con "grandi sacramentari” da alcuni decenni si designano varie importanti tradizioni eucologiche: cf Appendice I.

10 Per un primo orientamento cf Glossarium medice et infimce latinitatis, conditum a Carolo Dufresne, domino Du Cange, auctum a monachis ordinis S. Benedicti cum supplementis integris D.P. Carpenterii et additamentis Adelungii, et aliorum digessit G.A.L. Henschel (...), Paris, Firmin Didot: 1 A-B 1840, 2 C-D 1842, 3 E-K 1844, 4 L-O 1845, 5 P-R 1845, 6 S-Z 1846 (accessibile in <http://ducange.enc.sorbonne.fr/>; Thesaurus Linguae Latinae Editus auctoritate et consilio Academiarum quinque Germanicarum Berolinensis Gottingensis Lipsiensis Monacensis Vindobonensis, Lipsiæ, in ædibus Teubneri 1900-; A. Blaise, Le vocabulaire latin des principaux thèmes liturgiques. Ouvreage revu par A. Dumas, Brepols, Turnhout 1966; R.E. Latham et Al. (edd.), Dictionary of Medieval Latin Sources from British Sources, Oxford University Press for The British Academy, Oxford 1975-; A. Blaise, Dictionnaire latin-français des auteurs chretiens, revu specialement pour le vocabulaire théologique par H. Chirat, Brepols, Turnhout 1993.

11 È questo un terreno fecondo per la ricerca anche se talora si può giungere a conclusioni diametralmente opposte. Si pensi, ad esempio, alle relazioni tra le preghiere contenute nel Rotolo di Ravenna e le meditazioni omiletiche del vescovo ravennate san Pier Crisologo, relazioni giudicate appunto in modo opposto da A. Olivar (Los sermones de
} 
Soltanto poche fonti liturgiche sono state analizzate in modo sistematico con la redazione finale di un indice dei vocaboli o di una concordanza completa. Si tratta perlopiù di lavori editi in appendice ad alcune edizioni, di ricerche lessicografiche e riflessioni teologiche su singoli vocaboli ${ }^{12}$, oppure su espressioni più complesse ${ }^{13}$ : è un ricco materiale che meriterebbe tuttora di essere integrato con nuovi studi, alla luce di quanto segnalato nell'Appendice II alla presente Introduzione.

Una minima parte del thesaurus eucologico accumulato nei secoli è stata accolta nella redazione del messale della Curia romana (secolo XIII), l'antenato diretto del messale tridentino ${ }^{14}$. Su quest'ultima miniera di testi si è concentrata l'attenzione di liturgisti e filologi negli anni intorno al Concilio Vaticano $\mathrm{II}^{15}$. Le acquisizioni tecniche in ambito digitale hanno permesso di sistemare i dati che riguardano il messale in lingua latina ${ }^{16}$, le pubblicazioni liturgiche con elementi diversi dalle tradizionali orazioni presidenziali ${ }^{17}$, ad esempio i canti ${ }^{18}$. Grazie all'informatica si assiste ad un'innovazione radicale nell'elaborazione delle concordanze eucologiche. Esse non si limitano più a segnalare singoli vocaboli ma, come già avveniva in molte concordanze bibliche, inseriscono i singoli lemmi nel loro contesto; contesto, è bene ricordare, che spesso chiarifica il significato e la funzione del lemma stesso.

San Pedro Crisologo. Estudio critico = Scripta et Documenta 13, Abadia de Montserrat 1962), e da S. Benz (Der Rotulus von Ravenna nach seiner Herkunft und seiner Bedeutung für die Liturgiegeschichte kritish untersucht $=\mathrm{Li}$ turgiewissenschaftliche Quellen und Forschungen 45, Aschendorff, Münster 1967); replica di A. Olivar, Abermals der Rotulus von Ravenna, in Archiv für Liturgiewissenschaft 11 (1969) 40-58.

12 A questo proposito la bibliografia è molto vasta, cf Appendice II.

13 Esemplare è ancora il lavoro di G. Manz, Ausdrucksformen der lateinischen Liturgiesprache bis ins elfte Jahrhundert $=$ Texte und Arbeiten, 1. Abt., Beiheft 1, Erzabtei, Beuron 1941. Si tratta di un'indagine sistematica sul materiale eucologico dei sacramentari al fine di individuare gli elementi propri di ciascuna tradizione e rilevare la presenza di espressioni estranee (es. ispaniche) nelle fonti romane; sono prese in considerazione 1105 espressioni, da abiectio carnis a viventium omnium mater.

14 P. Bruylants, Les oraisons du Missel Romain. Texte et Histoire. I: Tabula synoptica fontium Missalis Romani. Indices. II: Orationum textus et usus juxta fontes = Etudes Liturgiques 1, Centre de Documentation et d'Information Liturgiques - Abbaye du Mont César, Louvain 1952. Nel I volume sono presentate - nei singoli formulari - le diverse tradizioni eucologiche testimoniate da una quarantina di fonti manoscritte e a stampa (edizioni del 1474, 1570 e 1604). Index verborum (incompleto): 217-281, Index orationum alfabetico: 283-330. Tabella sinottica delle domeniche dopo Pentecoste (16 testimoni): tra le pp. 56-57. Nel II volume per ogni orazione, disposta in ordine alfabetico, si fornisce un apparato critico testuale. Tutto questo materiale è rifluito nel volume Liturgia tridentina, cit. nella nota n. 1. - Molto utili sono le indagini come quella condotta da M.P. Ellebracht, Remarks on the Vocabulary of the Ancient Orations in the Missale Romanum = Latinitas Christianorum Primæva 18, Dekker - van de Vegt, Nijmegen - Utrecht 1963.

15 È pionieristico il lavoro di A. Pflieger, Liturgica orationis concordantia verbalia, cit. sopra nella nota n. 2.

16 M. Sodi - A. Toniolo, Concordantia et indices Missalis Romani (Editio typica tertia) = Monumenta Studia Instrumenta Liturgica 23, Lev, Città del Vaticano 2002. Il repertorio è accessibile in $<$ http://www.rifugiodelleanime. $\mathrm{org} / \mathrm{m} 3 />$. M. Sodi - A. Toniolo, Prcenotanda Missalis Romani. Textus - Concordantia - Appendices (Editio typica tertia) $=$ Monumenta Studia Instrumenta Liturgica 24, Lev, Città del Vaticano 2003.

17 Per le benedizioni episcopali cf E. Moeller, Corpus Benedictionum Pontificalium, I: Pars prima; II: Pars secunda; III: Concordantia verborum A-B; IV: Concordantia verborum $C-Z=$ Corpus Christianorum. Series Latina 162, 162A/C, Brepols, Turnholti 1971 e 1979.

18 Cf G. Milanese, Concordantia et instrumenta lexicographica ad GRADUALE ROMANUM pertinentia, præfata est M. Ferrari = Bibliotheca Gregoriana 1, Editrice Liguria, Genova - Savona 1996. Concordanze di tutte le parole presenti nei canti della Messa pubblicati sia nel Graduale Romanum del 1974 che nell'Antiphonale Missarum Sextuplex [n. 0902]. Tra gli strumenti lessicografici è da segnalare l'indice alfabetico retrogradus: 395-431. Nel lavoro inedito di S. Jung Kim - Le Sequenze nei cinque Graduali della Biblioteca Capitolare di Benevento. Trascrizione diplomatica comparativa, apparato critico e musicale, analisi. Indice delle concordanze verbali (tesi dottorale, dattilo) PIMS, Roma 1999 - è presentata l'edizione comparata di 130 pezzi; nello studio e negli apparati si tengono in considerazione anche testimoni non beneventani dell'Italia centrale. L'indice alfabetico delle concordanze verbali è nel vol. IV, pp. 1104-1363. 
Un rilancio del patrimonio letterario liturgico è avvenuto con la redazione della liturgia riformata in base agli orientamenti del Concilio Vaticano II sia per quanto riguarda la celebrazione eucaristica sia per ciò che concerne la preghiera delle ore. Importanti contributi stanno dischiudendo i particolari nascosti anche nelle traduzioni in lingue moderne ${ }^{19}$. Questa primavera della ricerca è annunciata da un rinnovato interesse per la lingua latina e per il significato pregnante di tante espressioni che nel tempo hanno offerto una ricca varietà di sfumature nel loro significato.

Tanto più urgente s'avverte la possibilità di disporre di nuovi strumenti di lavoro lessicografici che oggi possono aiutare l'impegno di studio soprattutto in tre ambiti:

- La conoscenza della spiritualità cristiana e del pensiero teologico quale si concretizza di generazione in generazione nell'esperienza delle Chiese locali, chiamate tutte a vivere coralmente un'unica fede in un reciproco arricchimento messo in evidenzia dalla storia particolare e dalla sensibilità peculiare di ogni tempo e luogo. Ė questo l'ambito più importante e anche più interessante che investe la redazione, prima, e poi la fruizione delle concordanze. Da strumento filologico la concordanza delle orazioni si fa strumento che permette di leggere la mappatura della preghiera personale negli imprevedibili itinerari dello Spirito.

- Le concordanze verbali sono tra gli strumenti più utili nel campo della ricerca codicologica che si propone di individuare la tradizione cui appartengono i molti libri e gli innumerevoli frammenti disseminati un po' ovunque. Frammenti che presentano varie difficoltà di lettura, a cominciare dallo stato pietoso in cui molti ancora si trovano. A questo aspetto s'aggiunge la difficoltà di individuare la natura di una miriade di pezzi acefali, per la cui identificazione servono poco o nulla i più diffusi indici alfabetici delle formule.

- Un settore in balia degli alti e bassi della presente infelice congiuntura culturale, è quello della filologia latina, sia classica sia medievale. È un forte segno di speranza il fatto che ci sia ancora qualche volenteroso (e insieme volontario) che s'occupa di questo settore da tanti irresponsabili ritenuto "morto". Strumenti come le concordanze sono, d'altra parte, uno stimolo per suscitare rinnovati interessi, sono un'occasione per scoprire i meandri dei labirinti verbali che la lingua latina aiuta a percorrere per raggiungere il senso profondo di quanto è stato esperito in quello spazio privilegiato che rimane tuttora culmen et fons della vita cristiana.

\section{Il sacramentario di Trento e la tradizione gregoriana}

Durante l'elaborazione del progetto per redigere una concordanza del sacramentario Gregoriano, tra le questioni primarie è stata affrontata la scelta del testo base. Tra le opzioni possibili si presentavano alcune edizioni moderne: dalla recensione di Cambrai $164^{20}$ a quella di Autun

19 Ad esempio, E. Mazza, Concordanze verbali delle collette e prefazi del messale romano italiano = Bibliotheca “Ephemerides Liturgicae”. Subsidia 39, Clv, Roma 1987, pp. 629.

20 J. Deshusses (ed.), Le sacramentaire Grégorien. Ses principales formes d'après les plus anciens manuscrits. Edition comparative. I: Le sacramentaire. Le supplément d'Aniane = Spicilegium Friburgense 16, Ed. Universitaires, Fribourg 1971. Edizione dei testi con apparato delle fonti parallele (compresi il veronese e i gelasiani) e apparato critico basato sui mss Autun, Bibl. Municipale, 19 (Marmoutier 845 circa); Cambrai, Bibl. Municipale, 162 e 163 (Saint-Vaast di Arras s. IX²); Donaueschingen, Hofbibl., 191 (Costanza s. IX 3/4); Düsseldorf, Landes- u. Stadtbibl., D1 (Essen s. IX 1/4); Firenze, Bibl. Med. Laurenziana, Edili 121 (Italia sett. s. IX ex-X in); Köln, Bibl. des Metropolitankapitels, 88 (Colonia [ed. Pamelio] sec. IX ex-X in); 137 (Colonia s. IX 2); Le Mans, Bibl. Municipale, 77 (Saint-Amand s. IX 3/4); London, British Library, Add. 16605 (Stavelot s. IX 2); Mainz, Seminarbibl., 1 (S. Albano di Magonza s. IX ex); Modena, Bibl. Capitolare, O.II.7 (Modena o Reggio Emilia s. IX m); Monza, Bibl. Capitolare, Sacramentario di Berengario, Francia sett. s. IX m); New York, Pierpont Morgan Libr., G 57 (Saint-Amand poi Chelles 860 c.); Oxford, Bodleian Libr., Auct. D.I.20 (San Gallo s. IX 2); Add. A 173 (Francia? s. IX m); Padova, Bibl. Capitolare, D 47 (Francia per l'Italia sett. s. IX 2/4 m); Paris, BNF, lat. 2290 (Saint-Amand s. IX 2); lat. 2292 (corte di Carlo il Calvo s. IX 
$19 \mathrm{bis}^{21}$ a quella del Paduense D $47^{22}$. Al termine di varie valutazioni, senza per nulla negare il valore di altri testimoni, si è data la preferenza al testo tramandato dal sacramentario di Trento ${ }^{23}$.

Il codice scritto a Trento, o in area alto-atesina per Trento, è oggi conservato nella località nativa ${ }^{24}$. Il sacramentario tridentino è un autorevole testimone di una tradizione italica dell'eucologia gregoriana anteriore al libro inviato da papa Adriano alla corte franca. Inoltre, nonostante alcune integrazioni di epoca successiva, il tridentino sembra rappresentare la recensione più arcaica del sacramentario gregoriano ${ }^{25}$.

\subsection{Osservazioni sul testo delle concordanze}

Il testo base utilizzato per le concordanze è quello dell'edizione $1985^{26}$, alla quale si rimanda per ogni particolarità ortografica del manoscritto. Quella edizione ha emendato il codice in più punti, rettificando errori e/o anomalie grafiche che in parte sono state segnalate in apparato ${ }^{27}$.

In vista della concordanza, si è ritenuto opportuno fare altri interventi sul testo al fine di rendere ottimale la serie alfabetica dei lemmi presi in considerazione. La " $e$ ", ad esempio, nel caso del dittongo (ae/oe) è stata pertanto resa sempre " $a e$ ". In presenza di lezioni differenti che interessano un unico e medesimo lemma, tutte le varianti sono state ricondotte alla lezione corretta o più frequente ${ }^{28}$. Così pure sono state uniformate alla lezione della prima parte quelle varianti che si trovano nella seconda, a partire da alcuni nomi propri ${ }^{29}$.

3/4); lat. 2812 (Lione s. IX 1); lat. 9429 (Francia sett. s. IX 2); lat. 12050 (Corbie 853); Sainte-Geneviève, 111 (Parigi s. IX 2); Reims, Bibl. Municipale, 213 (Saint-Amand 870 c.); Roma, BAV, Ottob. lat. 313 (cattedrale di Parigi s. IX 3/4); Regin. lat. 337 (Lione s. IX 1); Trento, Bibl. Provinciale d'Arte, 1590 (Trento s. IX 1); Verona, Bibl. Capitolare, XCI (Verona s. IX 1/4); LXXXVI (Verona s. IX 2/3); Wien, Österreichische Nationalbibl., lat. 1815 (Reichenau s. IX $m)$; Zürich, Zentralbibl., Rheinau 43 (Francia sett. s. IX ex).

21 J. Décréaux, Le sacramentaire de Marmoutier (Autun 19 bis) dans l'histoire des sacramentaires carolingiens du IXe siècle. I: Etude. Revue et mis au point par V. Saxer; II: Texte. Revue et mis au point par V. Saxer = Studi di antichità cristiana 38, Pontificio Istituto di Archeologia Cristiana, Città del Vaticano 1985.

22 A. Catella - F. Dell'Oro - A. Martini (edd.), adlaborante F. Crivello, Liber Sacramentorum Paduensis (Padova, Biblioteca Capitolare, cod. D 47) = Monumenta Italiæ Liturgica 3, Edizioni Liturgiche, Roma 2005, pp. 595.

23 Su varie iportesi relative alla natura del sacramentario, alla datazione e alla sua origine, cf lo studio esauriente di F. Dell'Oro in Monumenta Liturgica Ecclesice Tridentince sceculo XIII antiquiora. II A: Fontes liturgici. Libri sacramentorum. Studia et editionem paravit F. Dell'Oro, adlaborantibus B. Baroffio - I. Ferrari - H. Rogger, Società di Studi Trentini di Scienze Storiche, Trento 1985, pp. 18-47. Altri pochi interventi chiariscono alcuni particolari, ad es.: J. Deshusses, Le sacramentaire grégorien de Trente, in Revue Bénédictine 78 (1968) 261-282; K. Gamber, Der Codex Tridentinus (Ein Sakramentar der Domkirche von Säben aus der Zeit um 825), in Scriptorium 24 (1970) 293304 + pl. 17; F. Unterkircher, Das karolingische Sakramentar von Trient für Säben geschrieben?, in Der Schlern $51 / 1$ (1977) 54-60 + $10 \mathrm{Abb}$.

24 Già Trento, Biblioteca vescovile del Castello del Buonconsiglio, Cod. 217. Dal 1803 il manoscritto si trovava a Wien, Kaiserliche und Königliche Hofbibliothek (attuale Österreichische Nationalbibliothek), Cod. Vindobonensis 700; nel 1919 il libro è tornato a Trento ed è conservato oggi nel Castello del Buonconsiglio, Monumenti e Collezioni provinciali, 1590.

25 Cf Deshusses, Le sacramentaire Grégorien. Ses principales formes, pp. 71-72.

26 Monumenta Liturgica Ecclesice Tridentince. II A: Fontes liturgici, pp. 73-416. Il testo è stato stabilito da Dell'Oro; Baroffio ha collaborato per gli apparati. Le presenti concordanze interessano unicamente i testi eucologici del sacramentario, non i due elenchi dei Capitula e i pochi testi di letture e canti che sono inseriti in pochi formulari.

27 Ad esempio, nella formula n. 22 il ms ha pleps che nel testo è stato corretto in plebs; nella n. 44 il ms ha tua (moderamine) emendato in tuo. Su alcune particolarità grafiche di ordine grammaticale e fonetico cf Dell'Oro, Fontes liturgici, pp. 66-67.

28 Particolare è il caso del termine tecnico missa che talora è stato assunto con valore assoluto per cui si comprendono vari ad missa. Ciò nonostante si è intervenuto leggendo sempre ad missam; d'altra parte Incipiunt missas è stato reso con missae.

29 Che la seconda sezione del sacramentario abbia una storia pregressa differente dalla prima, si vede chiaramente anche dalla nomenclatura che in molti casi si discosta preferendo termini abituali nella tradizione gelasiana: secreta 


\subsection{La Concordantia}

Nella Concordanza del Sacramentario di Trento sono stati presi in esame i testi delle formule senza le ulteriori indicazioni specifiche, che riguardano l'uso della stesse.

La procedura KWIC in un'unica riga è stata scelta perché le formule di breve lunghezza sono molto numerose nei Sacramentari. Le occorrenze del termine sono presentate seguendo la numerazione progressiva delle formule del Sacramentario; si permette, così, di cogliere l'uso del termine all'interno delle singole sezioni del Sacramentario e si facilita uno studio più puntuale. Questo tipo di concordanza è definito KWIC, acronimo di key word in context, perché le parole oggetto di ricerca sono allineate e riportate con co-testo da entrambi i lati. L'allineamento scelto, in questo caso, è "centrato".

Ogni formula liturgica, di qualsiasi lunghezza essa sia, è stata considerata come campo di ricerca a sé stante. Il simbolo / presente nelle varie righe indica la separazione di una formula dalla successiva: il lettore è indirizzato verso l'inizio o la fine della formula stessa.

È stato necessario eliminare tutti gli accenti fonetici dalle vocali, e i dittonghi $(a e, o e)$ sono stati scritti per esteso; non sono stati eliminati i que. Non sono presenti le lettere alfabetiche $\mathrm{J}, \mathrm{K}, \mathrm{W}$; è presente invece la lettera $\ddot{\boldsymbol{e}}$ una volta nella parola aëris.

Per un ordinamento alfabetico corretto, tutte le lettere sono state considerate come minuscole anche se nella trascrizione definitiva il termine maiuscolo compare così come riportato nel testo.

Sono stati stabiliti 70 caratteri per ogni riga; le parole iniziali o conclusive della riga, spezzate da questa scelta, sono state automaticamente eliminate. In questo modo vi sono righe più lunghe o più brevi.

Il numero totale delle occorrenze del termine precede il termine stesso, messo in evidenza dal grassetto. All'interno della riga la parola cercata è evidenziata in grassetto, anche se ricorre più volte ma è computata una sola volta.

Sono stati esaminati 5966 vocaboli, per un complessivo conteggio di 36979 parole vagliate in totale.

È possibile scorrere i vari lemmi per cogliere il numero della specifica occorrenza; vari lemmi pur sempre segnalati non sono stati considerati data la non rilevanza ai fini dello studio dell'eucologia. In Appendice, infine, sono riportati i termini secondo il loro ordine di occorrenza, dalla congiunzione et che ricorre 1633 volte fino ai numerosissimi unicum.

\section{Il Sacramentario di Verona}

Nell'ottobre del 1713 avviene una scoperta di estremo interesse per la scienza liturgica e per la tradizione cultuale dell'Occidente. Il marchese veronese Scipione Maffei (1675-1755) trova un codice rimasto nascosto per secoli, insieme ad altri antichi e significativi manoscritti; ci si rende subito conto dell'importanza di questo unicum.

Il bisogno di conoscere il testo farà sì che durante quello stesso secolo XVIII avvengano ben quattro edizioni, fino alla quinta apparsa verso la fine del sec. XIX e poi alla sesta che costituisce la base dell'attuale concordanza ${ }^{30}$. Nell'edizione critica di Mohlberg il lettore può

e post communionem invece di super oblata e ad complendum. Per ragione di uniformità, alcuni lemmi sono stati modificati rispetto al testo originale; le lezioni sono segnalate in apparato.

30 La prima edizione fu fatta, d'accordo con il Maffei che dà il titolo di "veronense", dall'oratoriano Giuseppe Bianchini: Codex sacramentorum vetus Romanae Ecclesiae a sancto Leone papa I confectus [...], in Francsco Bianchini, Anastasii Bibliothecarii de vitis Romanorum Pontificum, vol. IV, Roma 1735; si tratta di un testo preciso 
trovare la descrizione dettagliata del manoscritto, le caratteristiche linguistiche del testo (ortografia, imprecisioni, grammatica, clausole), il contenuto e le caratteristiche del libro, i tentativi di datazione del Veronense (il Mohlberg ne enumera ben 85), il sacramentario in rapporto agli altri sacramentari latini, la descrizione delle prime edizioni, e i principi basilari dell'edizione stessa apparsa nel 1954.

A tre secoli esatti dalla scoperta del codice, è apparsa la nostra Concordantia. La coincidenza casuale si è accompagnata anche al $50^{\circ}$ della Sacrosanctum Concilium, in un felice incontro che ha permesso di far riecheggiare ancora una volta quanto la stessa Sacrosanctum Concilium affermava nel n. 23 a proposito dell'intimo rapporto che deve intercorrere fra tradizione e progresso nella riforma liturgica, da realizzare attraverso «un'accurata ricerca teologica, storica e pastorale».

\subsection{Il Sacramentarium}

Già attribuita a papa Leone Magno († 461), la raccolta eucologica conservata nella Biblioteca Capitolare di Verona (ms. LXXXV [80]), per alcuni secoli è stata denominata "sacramentario leoniano", mentre da pochi decenni si preferisce la denominazione neutrale "sacramentario veronese". Gli approfondimenti codicologici, storici e liturgici hanno permesso di valutare in modo più adeguato il libro che raccoglie oltre 1300 antiche orazioni liturgiche destinate alla celebrazione eucaristica ${ }^{31}$. Più che di un sacramentario in senso stretto, si tratta di una raccolta di libelli missarum, la più antica del genere, collezione preziosa per la conoscenza della liturgia dell'Urbe, del pensiero teologico e della lingua latina nella sua declinazione liturgica.

Sulla base dell'edizione del Sacramentarium Leonianum curata da Feltoe ${ }^{32}$, che sotto il profilo critico supera quelle precedenti del secolo XVIII, nell'immediato dopoguerra Placide Bruylants ha pubblicato una monumentale concordanza verbale del sacramentario veronese ${ }^{33}$. La moderna edizione, curata da L. C. Mohlberg ${ }^{34}$, ha stabilito un testo più critico con un minuzioso apparato filologico che aiuta a interpretare passi oscuri; non mancano interessanti congetture e interventi per ricostruire un testo attendibile ${ }^{35}$.

anche per le migliorie che vi si apportano. La seconda edizione è realizzata da Ludovico Antonio Muratori: Liturgia romana vetus, vol. I, Venezia, 1748; segue quindi l'edizione di Giuseppe Luigi Assemani: Codex liturgicus ecclesiae universae, vol. 6, Roma 1748; i due fratelli Pietro e Gerolamo Ballerini curano l'opera: S. Leoni Magni opera omnia, vol. II, Venezia 1756; finalmente Charles Lett Feltoe: Sacramentarium leonianum, Cambridge 1896.

31 Una nutrita e aggiornata bibliografia, ancora inedita, è stata redatta da Mons. Andrzej Suski, vescovo di Toruń in Polonia. Nel repertorio compaiono numerosi studi relativi a tematiche teologiche e a singoli vocaboli.

32 Charles Lett Feltoe, Sacramentarium Leonianum Edited, with Introduction, Notes and Three Photographs, Cambridge, Cambridge University Press 1896. Il volume è accessibile in http://archive.org/details/sacramentariuml00feltgoog

33 Placide Bruylants, Concordance verbale du Sacramentaire Léonien [ms. Vérone, Bibliothèque Capitulaire, LXXXV (80)], Louvain, Editions de l'Abbaye du Mont César s. d., pp. XVI + 697. Si tratta di un estratto della rivista “Archivum Latinitatis Medii Aevi” (Bulletin Du Cange) 18, 1945, 51-376 e 19, 1948, 39-405.

34 Sacramentarium Veronense (Cod. Bibl. Capit. Veron. LXXXV [80]). In Verbindung mit Leo Eizenhöfer OSB und Petrus Siffrin OSB herausgegeben von Leo Cunibert Mohlberg OSB, Roma, Herder 1954 (Rerum Ecclesiasticarum Documenta. Series maior. Fontes 1). Terza edizione rivista e aggiornata da Leo Eizenhöfer nel 1966, ristampa anastatica 1994.

35 Oltre alle note dell'apparato critico, cfr. in particolare la sezione $\S 2$ dell'introduzione a pp. XL-LIII "Die spraclichen Eigentümlichkeiten des Textes", dove sono esaminati l'ortografia, gli errori di scrittura e la grammatica. Sono segnalati, infine, vari studi sul cursus del testo. 
In vista della redazione della nostra concordanza, è stato necessario uniformare le differenti grafie del codice ${ }^{36}$ e normalizzare il testo secondo la grafia moderna della lingua latina ${ }^{37}$. In questa redazione sono state inserite le correzioni e le congetture dell'edizione Mohlberg $^{38}$, mentre tutti i lemmi originali del manoscritto veronese sono disponibili nel sito $w w w$.liturgia.it. La normalizzazione del testo si è resa necessaria per permettere l'uso della concordanza senza troppi ostacoli. Un esempio: chi volesse studiare gli aspetti ecclesiologici presenti nel Veronese, non troverebbe nulla se cercasse il lemma ecclesia, dal momento che la grafia costante del sacramentario è aeclesia/ae/am.

Scritto a Verona nella prima metà del VII secolo, il sacramentario veronese tramanda la più antica e ampia raccolta organica dell'eucologia romana. Come evidenziano anche le numerose emendazioni e integrazioni del testo originale ${ }^{39}$, il documento è in uno stato precario. La trasmissione "svogliata" ${ }^{40}$ e spesso corrotta delle orazioni ${ }^{41}$, non diminuisce, tuttavia, l'importanza dei testi trasmessi che riflettono uno stato arcaico della vita liturgica della Chiesa che è in Roma. Basta pensare, al riguardo, alle lezioni testuali alternative ${ }^{42}$, probabile eco di un rapporto particolare con i testi liturgici prima della loro rigida fissazione scritta. Soprattutto a riscuotere l'attenzione sono i molti testi che sembrano essere circoscritti a questo testimone, senza che se ne trovi traccia nelle tradizioni diffuse e affermatesi con il nome di gelasiana e gregoriana ${ }^{43}$.

La complessità della trasmissione testuale del Veronese emerge dal ricordato elenco dei lemmi nella grafia originale del manoscritto, presentato in due liste accessibili nel sito

\footnotetext{
36 Sono state uniformate grafie, ad esempio, come "adiuvari" e "adiubari”, "adiuvetur" e "adiiubetur", "evangelistae" e "evangeliste", "obtineat" e "optineat", "plebem" e "plevem", "votiva" e "votiba"; "affectum" e "adfectum"; "praesta" e "presta"; "implere" e "inplere"...

37 Cfr. i lemmi nel Dizionario Georges-Calonghi.

38 Le integrazioni degli editori sono state inserite in corsivo.

39 Nell'edizione Mohlberg sono indicati tutti gli interventi con cui nel tempo è stato emendato il testo, quasi sempre mediante la rasura di lettere e/o parole e la loro sostituzione. Quando è stato possibile, in apparato è stata proposta anche la lezione primitiva. Ad esempio, nel caso di "intuere" (orazione n. 10), si segnala che la " $u$ " è scritta sopra una lettera precedentemente erasa, forse una " $e$ ". Nella parola iniziale dell'orazione n. 23 "Sumentes", la seconda " $e$ " sostituisce la " $i$ " originale. - Tra gli interventi sono da ricordare le tante singole lettere e parole complete inserite negli spazi interlineari e nei margini. Nella presente edizione del testo normalizzato, tali lettere e parole sono scritte in neretto. Ad esempio, la parola corda dell'orazione 127. Dall'apparato si evince che si tratta di una correzione inserita di prima mano sul margine esterno per sostituire il termine peccata espunto nel testo e testimoniato anche dal Gelasiano Vaticano 1103. - Non mancano integrazioni posteriori come quella di una mano italiana che alla fine dell'ottavo secolo ha inserito dopo l'ultima riga di f. 24v (risulterebbe tra l'orazione 253 e il prefazio 254) un'altra orazione: Preces populi tui, quaesumus, domine, clementer adsume, ut nos servos tuos custodias ab omni malo adque defendas.

40 Tale svogliatezza è già stata notata poco dopo la redazione del codice e ha suscitato interventi correttivi. In molti casi gli emendamenti lasciano ancora riconoscere la lettera o le lettere erase e sostituite, tutte puntualmente segnalate nell'apparato dell'edizione Mohlberg. In molti casi, invece, si legge soltanto la sostituzione.

41 Un esempio dei tanti casi di corruzione segnalati in apparato riguarda il prefazio n. 244 . Il verbo consecrari o va emendato in consecrasti oppure va integrato con dignatus es. In alcuni casi, è il confronto con passi paralleli, dello stesso Veronese o di altre fonti eucologiche, che permette di risalire alla lezione corretta del testo. Nel prefazio n. 124, ad esempio, l'inizio Quorum martyrum è reso Quoniam martyrum grazie alla recensione presente nello stesso libro (nr. 410) e in altri sacramentari gelasiani, gregoriani e ambrosiani.

42 Seguendo l'edizione Mohlberg, le lezioni alternative sono pubblicate tra $|: x x x:|$. Nei casi in cui non sono indicate in quella edizione, ma sono segnalate come congettura nell'apparato, tali lezioni sono indicate con (|: xxx:|).

43 Negli ultimi anni, in alcuni casi isolati, sono stati individuati testimoni di testi ritenuti esclusivi del Veronese, in fonti eucologiche periferiche, a Piacenza e a Split (Spalato).
} 
$w w w$.liturgia.it. Una lista contiene i casi con peculiari grafie ${ }^{44}$, il secondo raccoglie i casi di corruzione o mende testuali ${ }^{45}$.

\subsection{Il testo base del sacramentario}

Il testo che costituisce la base su cui è stata poi fatta la concordanza è quello di Mohlberg. Intenso è stato il lavoro di revisione del testo latino in modo da fornire una versione facilmente usufruibile e corretta sotto l'aspetto linguistico.

In questa linea, oltre a quanto già rilevato, in grassetto sono poste le integrazioni interlineari e marginali; mentre in corsivo risultano le integrazioni all'edizione di Mohlberg.

Dal testo sono state tolte le abbreviazioni originali del codice, come pure tutte le dossologie delle orazioni e dei prefazi. Per una verifica completa è sempre doveroso fare riferimento all'edizione critica.

\subsection{La Concordantia e le Appendici}

Per formulare la concordanza del Sacramentarium Veronense che è composto da 139 pagine [ $(\mathrm{XX} \times \mathrm{x}$ fogli=160 pagine +1 singolo +1 doppio=163-24 (III x 8 fogli)=139], iniziando dal materiale eucologico utile per il 14 aprile, tra le varie pubblicazioni abbiamo scelto come base per la riformulazione l'edizione critica: Sacramentarium veronense, a cura di LEO CUNIBERT MOHLBERG OSB, Casa editrice Herder, Roma, 1956 (Series maior, Fontes I, RED)

Si contano 1331 pezzi ecologici ( + due con la dicitura bis), quindi 1333 in tutto tra orazioni e prefazi, raggruppati in formulari, composti di più pezzi eucologici.

Se si dovesse parlare di soli formulari la conta arriverebbe al n. 134 per 685 pezzi eucologici, difficilmente collocabili nella scansione annuale, che fa da supporto.

Vi sono formulari completi e formulari incompleti e spesso si incontra la sottotitolazione "ITEM ALIA", che potrebbe far presupporre un processo continuo di accumulazione.

È stato scelto, comunque, di mantenere i testi così come si presentano nella loro integrità testuale anche se ripetitivi o troppo simili: le formule non sono eccessive di numero e la lunghezza delle stesse è spesso breve.

Come per la precedente concordanza del Sacramentario gregoriano di Trento ${ }^{46}$ sono stati presi in esame i testi delle formule senza le ulteriori indicazioni specifiche, che potrebbero accompagnarle.

Ogni formula liturgica, di qualsiasi lunghezza essa sia, è stata considerata come campo di ricerca a sé stante. Il simbolo / presente nelle varie righe indica la separazione di una formula dalla successiva, indirizzando così l'occhio del lettore verso l'inizio o la fine della formula stessa.

\footnotetext{
44 In questa lista i lemmi sono disposti in ordine alfabetico: ac (= hac) 197 954, acerva 20, acervum 1216, Adauti 800 801, Adcumula 988, adcumulet 924 1217...

45 Nella seconda lista i lemmi sono disposti in ordine progressivo di orazioni. Nella prima colonna si propone il testo emendato dell'edizione Mohlberg; nella seconda la lezione originale, seguita dal numero dell'orazione.

innumerae in munere 11

confessione confessione ( $m$ espunto) 15

sustinere sustinuere 20

quoque quosque 22

46 Sacramentarium Gregorianum. Concordantia, a cura di Manlio Sodi - Giacomo Baroffio - Alessandro Toniolo, Las, Roma 2012.
} 
Nel testo della concordanza il numero totale delle occorrenze del termine precede il termine stesso, messo in evidenza dal grassetto.

Le occorrenze del termine sono presentate seguendo la numerazione progressiva delle formule del Sacramentario; si permette, così, di cogliere l'uso del termine all'interno delle singole sezioni del Sacramentario e si facilita uno studio più puntuale.

All'interno della riga la parola cercata è evidenziata in grassetto, anche se ricorre più volte, ma è computata una sola volta.

Sono stati stabiliti 70 caratteri per ogni riga; le parole iniziali o conclusive della riga, spezzate da questa scelta, sono state automaticamente eliminate. In questo modo vi sono righe più lunghe o più brevi.

Per un ordinamento alfabetico corretto, tutte le lettere sono state considerate come minuscole anche se nella trascrizione definitiva il termine maiuscolo compare così come riportato nel testo.

Anche per questo sacramentario si è scelta la forma più semplice di concordanza, definita $K W I C$, acronimo di key word in context, perché le parole oggetto di ricerca sono allineate e riportate con co-testo da entrambi i lati. L'allineamento scelto, anche in questo caso, è "centrato".

Sono stati esaminati 5966 tipi di termini rintracciabili in un vocabolario per un complessivo conteggio di 36979 parole vagliate in totale. Sono riportati, infine, i termini secondo il loro ordine di occorrenza; per l'ordine alfabetico si faccia riferimento al sito già sopra indicato. I più ricorrenti sono ut 893; et 1552.

Scorrendo la Concordanza il lettore troverà che per molti termini si dà solo il numero di occorrenze. Anche per non appesantire ulteriormente il volume, abbiamo ritenuto opportuno mantenere nel testo tutti quei termini che risultano utili ai fini di una ricerca filologica e teologica.

Nella prima Appendice è stato ritenuto opportuno riportare l'elenco alfabetico degli incipit di tutti gli embolismi prefaziali. Nella seconda, infine, si possono cogliere le occorrenze dei termini secondo il loro ordine.

\section{Il ,Reginensis 317“}

Tra i vari meriti che la regina Cristina di Svezia (Stoccolma 1626 - Roma 1689) può aver accumulato nella propria vita, gli studiosi del culto cristiano gliene riservano uno in particolare: quello di aver acquistato tra i tanti anche il codice del Sacramentarium Gelasianum, di averlo portato con sé a Roma dopo la sua conversione al cattolicesimo (1654), e soprattutto di averlo fatto giungere alla Biblioteca Apostolica Vaticana. Tutto questo è il frutto di una passione che ella aveva per i libri antichi e per i manoscritti, con l'intento di far diventare Stoccolma l'Atene del Nord!

È da questo fatto che il codice che contiene il sacramentario, insieme a numerosi altri, ha ricevuto il titolo di "reginensis" nel momento in cui fu acquisito dalla Biblioteca Vaticana ${ }^{47}$, dopo essere stato acquistato da Alessandro VIII con tutta la biblioteca alla morte della

47 Cf A. Wilmart, Codices Reginenses latini, vol. II, Biblioteca Apostolica Vaticana, Città del Vaticano 1945. 
Regina nel 1689. Nel frattempo il card. Giuseppe M. Tomasi avrà la possibilità di farne la prima edizione a stampa nel $1680^{48}$.

La paternità "gelasiana" è invece da ricondurre, secondo la maggior parte degli studiosi, a Valafrido Strabone ( $†$ 849) che lo "attribui”" a papa Gelasio (africano, 492-496) forse per dare maggiore autorità ai contenuti che sono di origine posteriore ai tempi di quel pontificato, anche se possiamo ritenere che una parte di libelli derivi da papa Gelasio, dal momento che una notizia nella Vita Gelasii del Liber Pontificalis afferma: «... fecit etiam et Sacramentorum praefationes et orationes cauto sermone...» ${ }^{49}$; e questo senza mettere in discussione l'opera di G. Pommarès ${ }^{50}$.

Al di là della "paternità", l'opera fu compilata nel sec. VIII vicino a Parigi (monastero di Chelles?), con elementi gallicani e soprattutto romani (questa l'opinione di J. Morin e di tanti altri studiosi dopo di lui); ma i contenuti risalgono fino al sec. VI. Per questo è denominato Sacramentarium Gelasianum Vetus per distinguerlo dagli omonimi rifacimenti databili al sec. VIII, frutti della fusione del Gelasiano con un Gregoriano Paduense. Il Gelasiano si presenta completo per tutte le celebrazioni dell'anno liturgico, dei sacramenti e per le varie circostanze; tutto il contenuto è articolato in tre libri. È d'importanza fondamentale per i numerosi testi che riguardano l'iniziazione cristiana, l'anno liturgico e altri sacramenti; ma anche per la ricchezza dei formulari per varie circostanze.

\subsection{Il Sacramentarium Gelasianum Vetus}

Il nucleo "gelasiano" è cronologicamente la seconda delle tre principali costellazioni eucologiche della Chiesa latina romana. All’Urbe sono collegati strettamente il più antico "sacramentario" veronese e la più recente raccolta gregoriana, mentre il repertorio gelasiano propone la tradizione romana integrata con materiale transalpino, fatto che si spiega con l'origine del manoscritto redatto in Francia.

Nelle altre aree dell'Europa latina medievale si affermano importanti tradizioni di cui restano isolati pochi testimoni come, ad esempio, il Missale Gothicum per la complessa realtà del mondo franco ${ }^{51}$.

Per individuare le peculiarità di una componente del patrimonio liturgico e, in particolare, di quello eucologico, non è sufficiente approfondire l'analisi delle poche fonti sopravvissute al tempo. Anzi, proprio l'esiguità "scandalosa" dei testimoni invita a fare studi con comparazioni

48 Cf Codices Sacramentorum nongentis annis vetustiores ... primum prodeunt cura et studio Ioseph Mariae Thomasij... Romae MDCLXXX. Il testo della Praefatio è apparso in traduzione italiana in Rivista Liturgica 101/3 (2014) 461-476. Il Tomasi all'inizio della sua opera così formula la dedica: «Quos Codices abhinc complura saecula conscriptos Munificentia Tua mihi concessit edendos: hos nunc fui foecundus exemplis Maiestati Tuae iure restituo. Qua in re utrumque gratulor, et sine prisci characteris molestia in hoc uno volumine eos lectitare Te posse: et de Re Sacra optime meritam ex eisdem apud eruditos omnes haberi. Accipe ergo quod praeclaram decet Sapientiam, Religionemque tuam: atque in eo summae observantiae in Te meae obsequium humaniter admitte. Vale». - Per un'adeguata presentazione della figura e dell'opera cfr. I. Scicolone, Il cardinale Giuseppe Tomasi di Lampedusa e gli inizi della scienza liturgica = Cultura cristiana di Sicilia 6, Palermo 1981; G.L. Masetti Zannini, Giuseppe Maria Tomasi, Cardinale santo e liturgista principe, Curia Generalizia dei Padri Teatini, Roma 1986; F. Andreu, Pellegrino alle sorgenti. S. Giuseppe Maria Tomasi. La vita - Il pensiero - Le opere, Curia Generalizia dei Chierici Regolari (Teatini), Roma 1987.

49 L. Duchesne, Liber Pontificalis, vol. I, 1955, p. 255.

50 Cf G. Pommarès (ed.), Gélase Ier: Lettre contre les lupercales et dix-huit messes du sacramentaire Léonien = Sources Chrétiennes 65, Cerf, Paris 1959.

51 Cf L.C. Mohlberg (ed.), Missale Gothicum (Vat. Reg. lat. 317) = Rerum Ecclesiasticarum Documenta, Series Maior, Fontes V, Herder, Roma 1961. 
eortologiche e confronti testuali tra le fonti dei vari ambiti. In queste ricerche - che ormai hanno alle spalle più di un secolo di laboriosa e proficua attività - i risultati sono talora sorprendenti, come quando Edmund Bishop ha rilevato i noti Spanish Symptoms presenti in aree lontane dalla penisola iberica, anche nella stessa area romana ${ }^{52}$.

Il lavoro che impegna varie discipline - dalla paleografia testuale a quella musicale, dalla storia delle istituzioni ecclesiastiche a quella della liturgia, dal culto dei santi alla filologia classica e mediolatina, dalla letteratura alla teologia e all'iconografia - è ancora lontano dall'aver raggiunto lo scopo, cioè la conoscenza adeguata dell'universo ecclesiale, quale si è cristallizzato nei testi dei sacramentari e degli altri libri liturgici.

\subsubsection{Disponibilità odierna di un'abbondante documentazione}

Questo lunghissimo e impervio cammino è oggi reso più facile da numerose edizioni critiche dei testi liturgici e da innumerevoli saggi e articoli. Un contributo particolare è fornito da sussidi specifici quali sono i repertori di carattere filologico-teologico. Essi hanno in Georg Manz un modello che attende prosecuzioni in varie direzioni ${ }^{53}$. Ci sono poi i repertori concentrati su un'unica fonte che producono concordanze di parole quanto mai utili54. Un'opera di imminente pubblicazione che solleciterà l'interesse di molti ricercatori è una Guida ai manoscritti di sacramentari e messali pretridentini di provenienza italiana ${ }^{55}$.

Il presente lavoro colma una grossa lacuna e rende disponibile l'elenco completo dei vocaboli che affiorano dai testi eucologici del sacramentario Gelasiano vetus ${ }^{56}$.

52 Cf E. Bishop, Spanish Symptoms, in Liturgica Historica. Papers on the Liturgy and Religious Life oft he Western Church, Clarendon Press, Oxford 1918, pp. 165-202 (lavoro pubblicato nel 1907).

53 Cf G. Manz, Ausdrucksformen der lateinischen Liturgiesprache bis ins elfte Jahrhundert = Texte und Arbeiten 1. Abt. Beiheft 1, Erzabtei, Beuron 1941. Si tratta di una indagine sistematica sul materiale eucologico dei sacramentari al fine di individuare gli elementi propri di ciascuna tradizione e rilevare la presenza di espressioni estranee (ad esempio, ispaniche) nelle fonti romane. Sono prese in considerazione 1105 espressioni da abiectio carnis a viventium omnium mater.

54 Cf C. Mohlberg - L. Eizenhöfer - P. Siffrin (edd.), Sacramentarium Veronense (Cod. Bibl. Capit. Veron. LXXXV [80]) $=$ Rerum Ecclesiasticarum Documenta, Series Maior, Fontes 1, Herder, Roma ${ }^{2} 1966$. Oltre alle 1331 formule del veronese, sono pubblicati il Rotolo di Ravenna: pp. 174-178 e p. 203; i Frammenti Milano, Bibl. Ambrosiana, O 210 sup., f. 46 (sec. VI-VII): pp. 178-180; i Frammenti Stuttgart, Württ. Landesbibl., H.B. VII 10 (sec. VII-VIII): pp. 180-181; Orazioni particolari del gelasiano del sec. VIII "Phillipps", Berlin, Staatsbibl., Phillipps 1667: pp. 182199; il Frammento Mone, Karlsruhe, Badische Landesbibl., Aug. CCLIII, f. 96v: pp. 200-201; i Frammenti anonimi ariani, Città del Vaticano, BAV, Vat. lat. 5750, f. 73-74: pp. 201-202. Indici delle citazioni e reminiscenze bibliche: pp. 230-234; vocaboli: pp. 235-438. - Cfr. inoltre per l'ambito ambrosiano: O. Heiming, Das Sacramentarium Triplex. Die Handschrift C 43 der Zentralbibliothek Zürich. I: Text; J. Frei, Das Sacramentarium Triplex. Die Handschrift C 43 der Zentralbibliothek Zürich. II: Wortschatz und Ausdrucksformen. Ein Wortverzeichnis = Liturgiewissenschftliche Quellen und Forschungen 49 = Corpus Ambrosiano-Liturgicum 1, Aschendorff, Münster W. 1968 e 1983. - Per i testi dei canti del Proprium Missae cfr. G. Milanese, Concordantia et instrumenta lexicographica ad Graduale Romanum pertinentia, praefata est M. Ferrari = Bibliotheca Gregoriana 1, Editrice Liguria, Genova - Savona 1996. Si tratta delle Concordanze di tutte le parole presenti nei canti della Messa pubblicati sia nel Graduale Romanum del 1974 sia nell'AMS [R.-J. Hesbert, Antiphonale Missarum Sextuplex d'après le graduel de Monza et les antiphonaires de Rheinau, du Mont-Blandin, de Compiègne, de Corbie et de Senlis, Vromant \& C, Bruxelles 1935]. Tra gli strumenti lessicografici da segnalare nella Concordantia c'è l'indice alfabetico retrogradus: pp. 395-431.

55 Si tratta dell'opera di G. Baroffio - M. Sodi - A. Suski, Sacramentari e messali pretridentini di provenienza italiana. Guida ai manoscritti, Società Bibliografica Toscana, Torrita (Siena) 2015 (in stampa).

56 Cf L.C. Mohlberg - L. Eizenhöfer - P. Siffrin (edd.), Liber Sacramentorum Romanae Aeclesiae ordinis anni circuli (Cod. Vat. Reg. lat. 316/Paris Bibl. Nat. 7193, 41/56) (Sacramentarium Gelasianum) = Rerum Ecclesiasticarum Documenta, Series Maior, Fontes 4, Herder, Roma ${ }^{3}$ 1981. Il manoscritto contiene 1704 formule. Nel volume sono inoltre pubblicati anche l'Exorcismus contra energumenos (nn. 1705-1725), lo Iudicium paenitentiale (nn. 1726-1786), il Breviarium Apostolorum... (nn. 1787-1799) provenienti dalla Bibl. Naz. di Parigi; inoltre i fram- 


\subsubsection{Testimone di peculiari tradizioni}

Il codice è praticamente l'unico testimone integro di una particolare tradizione eucologica che, come accennato, coniuga insieme le tradizioni romana e franca ${ }^{57}$.

Il nucleo centrale del sacramentario è stato attribuito a vari centri. Un'ipotesi ormai superata è stata proposta da Klaus Gamber, infaticabile ricercatore di nuove fonti liturgiche che interpretava con l'aiuto di una fantasia visionaria. A lui si deve la collocazione del Gelasiano a Ravenna ${ }^{58}$.

Oggi si è unanimi nel ritenere che si tratti sostanzialmente di un libro romano, ma divergono i giudizi sui particolari. Fa testo, quasi ovunque, una lunga ma non sempre solida narrazione pubblicata nel 1957 da Antoine Chavasse ${ }^{59}$. Tutti ripetono le sue mere ipotesi contrabbandate con il tempo fino a dichiararle e a ritenerle fatti reali, fuori discussione.

In questa situazione è difficile accettare nuovi scenari che susciterebbero un certo panico intellettuale; cadrebbero troppi castelli di carta in cui ci si rifugia per trovare conforto. Così l'acuto e tenace saggio di Massimo Martelli è stato ed è tuttora praticamente ignorato ${ }^{60}$.

menti "Bannister", Paris, BnF, lat. 10837, 42v-43r (Echternach sec. VIII/2, pp. 265-266) e i frammenti "Baumstark", London, British Libr., Add. 37518, pp. 116-117 (Inghilterra? Francia sett.? sec. VIII, pp. 266-267). - Per la bibliografia si può vedere l'ampia rassegna apparsa nella ristampa del 1981, alle pp. 325-330, oltre a quanto segnalato nella nota 13 .

57 Alcuni dati di carattere filologico emergono dagli elenchi e dagli apparati di opere quali P. Bruylants, Les oraisons du Missel Romain. Texte et Histoire. I: Tabulae synopticae fontium Missalis Romani. Indices. II: Orationum textus et usus juxta fontes = Etudes Liturgiques 1, Centre de Documentation et d'Information Liturgiques - Abbaye du Mont César, Louvain 1952. Nel I volume sono presentate - nei singoli formulari - le diverse tradizioni eucologiche testimoniate da una quarantina di fonti manoscritte e a stampa. Index verborum (incompleto): pp. 217-281, Index orationum alfabetico: pp. 283-330. L'opera è riprodotta in M. Sodi - A. Toniolo - P. Bruylants (edd.), Liturgia Tridentina. Fontes - Indices - Concordantia 1568-1962 = Monumenta Liturgica Piana 5, Lev, Città del Vaticano 2010. Inoltre: J. Deshusses - B. Darragon, Concordance et tableaux pour l'étude des grands sacramentaires, I: Concordance des pièces; II: Tableaux synoptique; III: Concordance verbale A-D; IV: Concordance verbale E- $L$; V: Concordance verbale $M-P$; VI: Concordance verbale $Q-Z=$ Spicilegii Friburgensis Subsidia 9-14, Ed. Universitaires, Fribourg 1982 [I-III] e 1983 [IV-VI]. Sono elaborate tutte le orazioni presenti nei "grandi sacramentari": veronese (o leoniano), gelasiano, gregoriano adrianeo, supplemento di Aniano, gelasiani del sec. VIII (Gellone, Angoulême, San Gallo 348). Il vol. I contiene l'indice alfabetico di tutte le orazioni; il vol. II presenta un prospetto di tutti i formulari e delle singole formule (4258) secondo la successione che essi hanno in ciascuna fonte; i voll. III-VI contengono le concordanze verbali di tutte le parole presenti in tutte le formule. - B. Coppieters't Wallant, Corpus Orationum, inchoante E. Moeller, subsequente I.M. Clément, 14 voll. = Corpus Christianorum, Series Latina 160 A-M, 161, Brepols, Turnholti 1992-2004. Nei primi 9 volumi sono pubblicate in ordine alfabetico e con apparato di varianti tutte le orazioni della Messa edite in molte pubblicazioni moderne.

58 Cf K. Gamber, Codices Liturgici Latini Antiquiores = Spicilegii Friburgensis Subsidia 1, Universitätsverlag, Freiburg ${ }^{2} 1968$, pp. 299-318; sui Gelasiani del sec. VIII cfr. pp. 368-397.

59 Cf A. Chavasse, Le sacramentaire Gélasien (Vaticanus Reginensis 316). Sacramentaire presbytéral en usage dans les titres romains au VIIe siècle = Bibliothèque de théologie IV: Histoire de la théologie 1, Declée \& Cie, Paris... 1958.

60 Sull'ipotesi della super sindonem nella liturgia romana cf A.M. Martelli, Un fenomeno della liturgia gallicana $e$ del Gelasiano: le messe con più orazioni prima della segreta, in Studia Patavina 19 (1972) 539-579= A.M. Martelli, Contributi allo studio del Sacramentario Gelasiano Reg. 316 (Nuove prospettive per la spiegazione della sua formazione). Estratto della Tesi di Laurea nella Facoltà Teologica del Pontificio Ateneo di S. Anselmo in Roma, Padova 1973; A.M. Martelli, Un fenomeno della liturgia gallicana e del gelasiano: le messe con più orazioni prima della segreta, in Studia Patavina 20 (1973) 546-569; A.M. Martelli, Il Sacramentario gelasiano. Cod. Vat. Reginense 316. Primo testimone completo dell'esperimento della Liturgia Romana nella Gallia Precarolingia, Vita Trentina Editrice, Trento 2003. 


\subsubsection{Dai Gelasiani del sec. VIII alla tradizione gregoriana}

Un problema non irrilevante è costituito dal complesso agglomerato gelasiano che vede raccogliersi intorno al Vetus un'ampia corona di satelliti: i gelasiani dell'VIII secolo o gelasiani di ultima generazione (Junggelasiana) prima dell'integrazione con la tradizione gregoriana e la formazione dei libri misti.

Questi sacramentari sono compilazioni frutto di "une réorganisation, effectuée à partir d'organisation antérieures" ${ }^{\prime \prime 1}$. Il problema sta nell'identificare queste fonti e i procedimenti redazionali. Anche in questo caso, le analisi filologiche di Bernard Moreton non hanno trovato un'adeguata risonanza e neppure l'elasticità mentale necessaria per aprirsi a orizzonti storici imprevisti, ma certo non nuovi ${ }^{62}$.

Basti considerare che la "redazione gelasiana, cui attingono direttamente i Gelasiani del sec. VIII, in alcuni casi è anteriore alla stessa redazione del Veronese o almeno di qualche libello di questa classica raccolta". Moreton, inoltre, prospetta che la redazione dei Gelasiani "sia da ricercarsi in un centro benedettino della zona vicina alle Alpi retiche verso il terzo quarto del sec. VIII. Di qui il Sacramentario si sarebbe diffuso presto nelle varie regioni" ${ }^{\prime 3}$.

Questi pochi accenni lasciano intravvedere l'importanza di sussidi come la concordanza qui pubblicata. Certamente nella storia delle tradizioni eucologiche (romane) è necessario studiare e confrontare i testimoni nella loro integrità, nella complessa articolazione delle sezioni e dei formulari, nella redazione delle singole orazioni e testi diversi (non si possono dimenticare neppure le rubriche).

Ma è indispensabile e urgente riprendere le analisi comparative anche sul piano lessicografico al fine di individuare le diverse stratificazioni dei libri liturgici, stratificazioni che emergono dalle modifiche semantiche e dall'uso di particolari vocaboli propri di determinate aree culturali e linguistiche e di precisi momenti cronologici.

\subsection{Il testo base del sacramentario}

Il testo che costituisce la base del presente lavoro è quello di Mohlberg, già citato. La sua edizione critica offre il punto di partenza non per riprodurre quanto già fatto, ma per offrire un testo in una forma latina per quanto possibile corretta. Solo così è possibile procedere ad un lavoro di concordanza tale da poter dialogare sia con altre fonti del primo millennio che soprattutto con quelle odierne.

\footnotetext{
${ }^{61}$ A. Chavasse, Le sacramentaire dans le group dit «Gélasiens du VIIIe siècle». Une compilation raisonnée. Etudes des procédés de confection et Synoptique nouveau modèle, I: Etudes particulières; II: Synoptiques et tableaux speciaux $=$ Instrumenta patristica 14 A/B, Abbatia S. Petri - M. Nijhoff, Steenbrvgis - Hagae 1984; citazione dal vol. I, p. iii. Cf M. Klöckener, Sakramentarstudien zwischen Fortschrift und Sackgasse. Entschlüsselung und Würdigung des zusammenfassenden Werkes von Antoine Chavasse über die Gelasiana des 8. Jahrhunderts, in Archiv für Liturgiewissenschaft 32 (1990) 207-230.

62 Cf B. Moreton, The Eight-Century Gelasian Sacramentary. A Study in Tradition = Oxford Theological Monographs, Oxford University Press, Oxford 1976. Un importante caso anteriore alle raccolte gelasiane - che è venuto in mente a Giacomo Baroffio quando nel 1976-1977 ha letto per la prima volta il saggio di Moreton - è quello della tradizione letteraria del Padre nostro tramandato nei Vangeli di Matteo e di Luca. Secondo Joachim Jeremias, la redazione più lunga, in quanto a estensione, è più recente, ha introdotto nuove formule; ma in quanto al vocabolario e al suo uso riflette la redazione primitiva.

63 Citazione dalla recensione di G. Baroffio (B.B.) in Rivista Liturgica 66/5 (1979) 714-716: 715; scheda 21 del Bollettino bibliografico.
} 
Come per il sacramentario Gregoriano e per quello di Verona ${ }^{64}$, il lavoro più impegnativo è consistito nel dare una forma corretta, sotto l'aspetto linguistico, a tutte le formule proprie del Gelasiano.

Non sono state considerate le formule presenti nell'edizione critica di Mohlberg collocate al termine del sacramentario stesso, fermandoci alla formula n. 1704, completata dall'explicit dell'opera.

La rilettura critica di tutto il testo ha permesso di uniformare varie grafie e talvolta di interpretare alcune espressioni. Ed è da questa prospettiva di un latino corretto che si può accedere allo studio filologico dei termini dialogando sia con i due precedenti sacramentari e sia con il Missale Romanum edito in seguito al Concilio di Trento e con quello edito per volontà del Concilio Vaticano $\mathrm{II}^{65}$.

Accostare in dettaglio l'intero Gelasiano, come già il Gregoriano e il Veronense, offre l'opportunità per cogliere non solo la ricchezza dei testi, ma anche e soprattutto l'abbondanza di prospettive teologico-liturgiche che solo un'attenta "meditazione" del testo può permettere. Quando poi - quod faxit Deus! - si potranno gustare le ricchezze dei tre più antichi sacramentari della tradizione occidentale anche in lingua viva, allora questo lavoro delle Concordantiae sarà apparso meno arido di quanto di fatto può sembrare.

Tra le tante sorprese che l'attento studioso può cogliere c'è anche il testo n. 312 che contiene la traditio Symboli con la traslitterazione interlineare del greco. Sembra essere il documento più antico che testimonia la traslitterazione del testo greco quando ormai non si celebrava più a Roma in questa lingua ${ }^{66}$.

Il testo base permette dunque di accostare anche questo sacramentario secondo l'ordine originario delle formule; ma permette anche di leggere con maggior attenzione il testo che è stato riprodotto per ordine di Paolo VI nel 197567: opera che corona quanto E. Burque aveva scritto: «... un des plus précieux documents liturgiques que la Providence nous ait conservé. C'est grâce à lui... qu'on est enfin arrivé à voir un peu clair dans l'histoire liturgique prégrégorienne et à résoudre des problèmes... qui autrement seraient demeurés d'éternelles énigmes.... ${ }^{68}$.

${ }^{64}$ Cf le due precedenti concordanze, sempre a cura di M. Sodi - G. Baroffio - A. Toniolo, Sacramentarium Gregorianum. Concordantia $=$ Veterum et Coaevorum Sapientia [= VCS] 7, Las, Roma 2012; Sacramentarium Veronense. Concordantia = VCS 10, Las, Roma 2013.

${ }_{65} \mathrm{Cf}$ al riguardo le due concordanze edite a cura di M. Sodi - A. Toniolo, Liturgia Tridentina ... sopra citata nella nota 10; e Concordantia et Indices Missalis Romani. Editio typica tertia = Monumenta Studia Instrumenta Liturgica 23, Lev, Città del Vaticano 2002.

66 Nella Concordantia non sono stati presi in considerazione i termini greci traslitterati in quanto sarebbe risultato un lavoro inutile.

67 Cf Sacramentarium Gelasianum e Codice Vaticano Reginensi Latino 316 vertente anno sacro MCMLXXV iussu Pauli PP. VI phototypice editum = Codices e Vaticanis selecti..., vol. XXXVIII, In Civitate Vaticana MCMLXXV. Il volume che accompagna l'edizione contiene due studi: L. Michelini Tocci, Il manoscritto [storia, scrittura, ornamentazione, descrizione], pp. 3-26; B. Neunheuser, Il «Sacramentario Gelasiano» (Reg. lat. 316) e la sua importanza per la storia della liturgia, pp. 27-46. Il testo di Michelini Tocci è stato ripreso e pubblicato in Rivista Liturgica 101/3 (2014) 477-500.

68 E. Bourque, Étude sur les sacramentaires romains. Première partie: Les textes primitifs = Studi di antichità cristiana 20, Pontificio Istituto di Archeologia Cristiana, Roma 1948, p. 187. 


\subsection{La Concordantia e le Appendici in internet}

Per la concordanza del Sacramentario, come già segnalato, abbiamo scelto come punto di riferimento l'edizione critica curata da L.C. Mohlberg - L. Eizenhöfer - P. Siffrin. Il Cod. Vat. Reg. Lat. 316 conta 245 fogli numerati progressivamente a partire dal $3^{\circ}$ fino al $245^{\circ}$ (nn. 1-1704). La parte finale del codice, che non è inclusa nella nostra pubblicazione, corrisponde ai ff. 41-56 del codice latino 7193 (nn. 1705-1799).

I 1704 pezzi eucologici, organizzati in formulari secondo un piano ben definito diviso in tre libri, contengono il "temporale" (nn. 1-803); il "santorale" (nn. 804-1177); e i testi "fuori calendario" (nn. 1178-1704).

Il Sacramentario Gelasiano è un libro specificamente approntato per gli interventi ministeriali e indica sia le azioni che i ministri devono compiere, sia le parole che devono accompagnare la gestualità liturgica. I brani contenuti si presentano, quindi, sotto forma di testi, trascritti per intero, che, da un lato, descrivono il comportamento dei ministri e dall'altro riproducono le parole proprie di questi ultimi.

Nel processo di "modellizzazione" per redigere la concordanza sono stati eliminati tutti i termini relativi ai gesti, che nel testo pubblicato sono evidenziati con la sottolineatura (e che corrispondono alle "rubriche"). Così pure non sono stati presi in considerazione i titoli dei formulari.

I testi eucologici delle formule sono stati mantenuti così come si presentano nella loro integrità e, come per le precedenti concordanze del Sacramentario Gregoriano di Trento e del Sacramentario Veronense, sono state eliminate le ulteriori indicazioni specifiche che le accompagnano.

Ripetendo quanto già scritto in precedenti edizioni di concordanze, ogni formula liturgica, di qualsiasi lunghezza essa sia, è stata considerata come campo di ricerca a sé stante. Il simbolo "/" presente nelle varie righe indica la separazione di una formula dalla successiva, indirizzando così l'occhio del lettore verso l'inizio o la fine della formula stessa.

Nel testo della concordanza il numero totale delle occorrenze del termine precede il termine stesso, messo in evidenza dal grassetto. Le occorrenze sono presentate seguendo la numerazione progressiva delle formule del Sacramentario; si permette, così, di cogliere l'uso del termine all'interno delle singole sezioni del Sacramentario e si facilita uno studio più puntuale.

All'interno della riga la parola cercata è evidenziata in grassetto e, anche se ricorre più volte, è computata una sola volta.

Dato il numero superiore delle formule del Gelasiano rispetto ai due precedenti sacramentari, per non appesantire il testo cartaceo, sono stati stabiliti 65 caratteri per ogni riga; le parole iniziali o conclusive della riga, spezzate da questa scelta, sono state automaticamente eliminate. In questo modo vi sono righe più lunghe o più brevi.

Per un ordinamento alfabetico corretto, tutte le lettere sono state considerate come minuscole anche se nella trascrizione definitiva il termine maiuscolo compare così come riportato nel testo.

Anche per questo sacramentario si è scelta la forma più semplice di concordanza, definita $K W I C$ perché le parole oggetto di ricerca sono allineate e riportate con co-testo da entrambi i lati. L'allineamento scelto, anche in questo caso, è "centrato". 
Scorrendo la Concordanza il lettore troverà che per molti termini si dà solo il numero di occorrenze; per non gravare il volume, abbiamo ritenuto opportuno mantenere nel testo tutti quei termini che risultano utili ai fini di una ricerca filologica e teologica. Tutti gli altri, comunque (siamo consapevoli che ogni selezione è sempre discutibile!), sono disponibili nel sito www.liturgia.it.

\section{Conclusione: Per philologiam ad theologiam}

La trilogia delle concordanze non ha altro obiettivo se non quello di facilitare l'accostamento dei tre più antichi sacramentari che hanno poi permesso di riversare tanta della loro ricchezza nel Missale Romanae Curiae ${ }^{69}$, nel Missale Romanum edito in seguito a quanto stabilito dal Concilio di Trento ${ }^{70}$, e soprattutto nel Missale Romanum (ma anche in altri libri liturgici) la cui rielaborazione è stata voluta dai Padri del Concilio Vaticano II ${ }^{71}$.

Una simile trilogia può avere più esiti nella sua valorizzazione. Lo studioso sa però che l'obiettivo principale di tutto questo è costituito dallo studio filologico per cogliere la ricchezza teologica dei termini propri dell'eucologia. Una ricchezza che è possibile approfondire quando si valorizza la filologia e l'analisi sintagmatica per giungere - in dialogo con tutte le altre espressioni parallele - a evidenziare lo specifico teologico di un termine sempre considerato nell'insieme della formula cui appartiene e più ancora del formulario e del tempo liturgico per cui è stato pensato, scritto e pregato.

È la metodologia liturgica - e più in generale quella teologica "sacramentaria" - che viene a beneficiare di simili strumenti di lavoro. La storia dell'ermeneutica specificamente liturgica ha fatto grandi progressi in tempi recenti; un'ermeneutica che tocca tutti i linguaggi del culto cristiano, ma in particolare quella relativa ai testi eucologici.

L'intreccio tra l'ermeneutica liturgica e quella biblica ha riaperto un'attenzione più spiccata al rapporto tra bibbia e liturgia ${ }^{72}$, facendo riemergere una "pagina" ben conosciuta al tempo dei Padri, e oggi quanto mai accolta come garanzia di ulteriori approfondimenti in vista di una teologia e spiritualità liturgica che nascono dall'esperienza del culto cristiano e ad essa riconducono anche attraverso lo studio filologico e testuale ${ }^{73}$.

I motivi per cui ci si può accostare al Missale Romanum possono essere i più diversi. Qualora, però, si voglia entrare nel merito dei significati dei singoli termini, dei sintagmi cui questi appartengono, della formula in cui si trova il sintagma... allora il ricorso ad uno strumento di confronto è indispensabile, in modo da verificare le diverse accezioni semantiche che uno stesso termine assume all'interno della medesima formula, del formulario, del

69 Cf R. Lippe (ed.), Missale Romanum. Mediolani 1474, I.: Texte = Henry Bradshaw Society 17, London 1907.

70 Cf M. Sodi - A.M. Triacca (edd.), Missale Romanum. Editio princeps (1570) = Monumenta Liturgica Concilii Tridentini 2, Lev, Città del Vaticano ${ }^{2} 2012$.

71 Per il Missale Romanum del Vaticano II e per una panoramica completa dei libri liturgici cfr. Rivista Liturgica 90/4 (2003) Missale Romanum. La «novitas» della terza edizione latina; 95/5 (2008) La liturgia di rito romano e i suoi libri; 97/3 (2010) La tradizione liturgica della Chiesa di Roma; e 98/3 (2011) Ermeneutica del libro liturgico.

72 Cf al riguardo il prezioso contributo di A.M. Triacca, Bibbia e liturgia, in D. Sartore - A.M. Triacca - C. Cibien (edd.), Dizionario di liturgia, San Paolo, Cinisello B. (Mi) 2001, s.v.

73 Due esempi recenti sono apparsi nella rivista Latinitas NS: M. Sodi, Latinitas liturgica. Una pagina esemplare circa il rapporto tra Scrittura ed eucologia, 1, 2013, 51-72; F.M. Arocena, Analecta hymnica Ecclesiae: himnarios e himnos, 2, 2014, 69-82. 
periodo liturgico, ecc. ${ }^{74}$ Solo un esame filologico-sintagmatico permette di cogliere i contenuti racchiusi nella scelta di un determinato termine a differenza di un altro.

L'attualità della Concordantia risulta dal fatto che essa permette di valorizzare un ricchissimo patrimonio difficilmente poco fruibile nella sua ricchezza; di confrontare quanto racchiuso, ad esempio, nell'odierna edizione del Missale con ciò che era presente nel Missale tridentino; di esaminare a fondo le scelte operate nella selezione dei testi (sia in quelle che non sono state fatte ma che era opportuno compiere, sia in altre che risulteranno superflue o fuorvianti rispetto alla tradizione); di compiere un lavoro di traduzione nelle lingue vive, verificando l'uso dello stesso termine in contesti diversificati.

In definitiva, solo uno strumento di questo genere permette di cogliere l'unità e valorizzare la ricchezza biblico-teologico-liturgica presente nei testi per la celebrazione dell'Eucaristia e degli altri sacramenti secondo il rito romano. È l'augurio che facciamo a tutti coloro che si confronteranno con queste pagine.

In questa ottica anche l'aridità di una concordanza può offrire un contributo prezioso per cogliere aspetti sempre nuovi della ricchezza teologica che promana dalla celebrazione dei santi misteri, e - di riflesso - dalla ricchezza della parola di Dio di cui gli autori delle formule avevano una consuetudine così intensa da offrirci testi perenni perché sempre attuali ${ }^{75}$.

\section{„Concordantia” of three great Sacramentary: Gregorian, of Verona and Gelasian Summary}

The "tradition" of the Church always amazes us with its wealth and the characteristics of its documents. Also the liturgical tradition contributes in this in the important way. It is important to find the appropriate tools to learn about the same documents. In this way the great work done by the three scholars of liturgy is an example and reminder. Looking through all of the text reveals the infinity of elements which doubtless can be a great help per philolgiam ad theologia or ad culturam!

Keywords: Concordantia, Sacramentary, Church

\section{„Concordantia” trzech wielkich Sakramentarzy: Gregoriańskiego, z Werony i Gelazjańskeigo \\ Abstrakt}

"Tradycja” Kościoła nigdy nie przestanie zaskakiwać nas swoim bogactwem i charakterystyką dokumentów. Również tradycja liturgiczna ma w tym swój znaczny wkład. Ważne jest znalezienie odpowiednich narzędzi, które umożliwią lepsze poznanie tych samych dokumentów. W tym przypadku dzieło napisane przez trzech badaczy z zakresu liturgiki stanowi

\footnotetext{
74 Si veda, ad esempio, il tipo di analisi dei testi eucologici che è possibile realizzare in vista della individuazione dei contenuti, nell'ampio studio di M. Sodi, La "latinitas" dei libri liturgici. L'eucologia del "Tempus Adventus" nel "Missale Romanum" di Paolo VI: dalla filologia alla teologia eucaristica, in E. dal Covolo - M. Sodi (edd.), Il latino e i cristiani. Un bilancio all'inizio del terzo millennio = Monumenta Studia Instrumenta Liturgica 17, Lev, Città del Vaticano 2002, pp. 375-488 (con ampia bibliografia).

75 Cf M. Sodi - M. Maritano (edd.), Leone I e Gregorio I. Attualità di due "grandi" promotori di cultura. Presentazione di E. Prinzivalli = Vivae Voces 29, Lateran University Press, Città del Vaticano 2015.
} 
przykład i wezwanie. Analizując tekst stwierdza się, że zawiera on wiele elementów, które bez wątpienia mogą stanowić wielką pomoc per philolgiam ad theologia lub ad culturam!

Słowa kluczowe: „Concordantia”, Sakramentarz, Kościół

Nota o Autorze: ks. prof. Manlio Sodi jest emerytowanym profesorem Università Pontificia Salesiana; jego dorobek naukowy obejmuje publikacje z zakresu historii i liturgiki; przez dwadzieścia lat był redaktorem czasopisma Liturgica.

Nota o Autorze: prof. Giacomo Baroffio jest emerytowanym profesorem muzykologii średniowiecznej Universita di Pavia; aktualnie prowadzi badania w zakresie historii tekstów muzycznych z pierwszego tysiąclecia.

Nota o Autorze: dr Alessandro Toniolo obecnie prowadzi badania nad źródłowymi tekstami liturgicznymi. 